Evans on the basis of five values lying within a range of $0 \cdot 3$. Deb's values for this vary from $1451 \cdot 6$ to $1459 \cdot 5$, with a mean of $1455 \cdot 5$. It is clearly essential in work of this kind, first to obtain a homogeneous set of data of the highest possible accuracy, and secondly to accept as significant only those intervals which agree within the order of accuracy of the data. The work under consideration fulfils neither of these requirements.

Again, the evidence obtainable from hyperfine structure work ${ }^{6}$ is entirely neglected by Deb, and in fact is only in occasional agreement with his allocations of $j$ values, although it is in harmony with Evans's observations.

Finally, Deb's value for the ionisation potential, $10 \cdot 548$ volts, is open to serious question. It is based on a very doubtful interpretation of an emission continuum with a maximum at $3406 \mathrm{~A}$.; this is presumably that commonly known by the wavelength $3460 \mathrm{~A}$. of its less refrangible edge. $\mathrm{He}$ attributes it on very slender evidence to the capture of an electron by an iodine atom in the $5 p^{4} .6 s .{ }^{2} P_{3^{\prime} 2}$ state. The evidence against this interpretation need not be detailed here, but it is of a very convincing character. Although several Rydberg sequences are given they do not lead as usual to independent values of the ionisation potential, since the term values involved are based on the above assumption; neither can they be regarded, in view of various objections to them which can be raised, as affording any support to that assumption.

In view of these criticisms I am bound to say that our knowledge of the structure of the iodine spectrum does not appear to have been sensibly advanced by the paper under consideration.

Armstrong College, W. E. Curtis.

Newcastle-upon-Tyne. Feb. 27.

1 Turner, Phys. Rev., 27, 397 ; 1926.

a Eans, Proc. Roy. Soc., A, 133, 417 ; 1931

3 Deb, Proc. Roy. Soc., A, 139, 380; 1933.

- Bloch, Ann. Phys., 11, 141; 1929.

5 Wood and Kimura, Astrophys. J., 46, 181 ; 1917.

- Tolansky, Proc. Roy. Soc., A., 136, 585 ; 1932.

\section{Line Absorption of Chromic Salts in Relation to Co-ordination}

THE absorption spectra of salts of trivalent chromium are characterised by exceedingly narrow bands in the red part of the spectrum. Their presence in the spectra of co-ordination compounds of chromium was discovered by Lapraik ${ }^{1}$ in 1890 , whose work is the more remarkable for being completed prior to Werner's theory of co-ordination valency and therefore at a time when the peculiar structure of the compounds was unknown.

We have recently examined the aqueous solutions of a number of chromic compounds by means of a spectrometer and have noticed a very simple fact which would doubtless have been missed had we used a spectrograph. In the case of every compound studied, only one narrow band is sufficiently strong to be detected by visual observation of dilute solutions in short observation tubes. In many cases it is as persistent as the neighbouring broad 'molecular' absorption band, for on dilution both disappear at about the same concentration. For convenience we shall refer to this narrow band as the 'characteristic band' of the compound.

An interesting relationship has been established between the chemical constitution of a complex ion and the position of the characteristic band. It is evident from the accompanying table that as the three electrically neutral molecules of ethylene diamine (en) in $\left[\mathrm{Cr}(\mathrm{en})_{3}\right]+++$ are successively replaced by negatively charged oxalate radicles, the band moves farther into the red. The position of the characteristic band of a complex ion is sensibly unaffected by the accompanying anions or cations.

\begin{tabular}{|c|c|}
\hline Molecular Ion & $\begin{array}{c}\text { Approximate Position of } \\
\text { Characteristic Band. }\end{array}$ \\
\hline$\left[\mathrm{Cr}(\mathrm{en})_{3}\right]+++$ & $6705 \mathrm{~A}$ \\
{$\left[\mathrm{Cr}(\mathrm{en})_{2} \mathrm{C}_{2} \mathrm{O}_{4}\right]_{+}$} & $6845 \mathrm{~A}$ \\
{$\left[\mathrm{Cr}(\mathrm{en})_{(2}\left(\mathrm{C}_{2} \mathrm{O}_{4}\right]-\right.$} & $6915 \mathrm{~A}$ \\
{$\left[\mathrm{Cr}\left(\mathrm{C}_{2} \mathrm{O}_{4}\right)_{3}\right]-+$} & $6970 \mathrm{~A}$ \\
\hline$\left[\mathrm{Cr}\left(\mathrm{H}_{2} \mathrm{O}\right)_{8}\right]+++$ & $6695 \mathrm{~A}$. \\
\hline
\end{tabular}

The condition of chromic ion in violet solutions of the simple salts is, presumably, $\left[\mathrm{Cr}\left(\mathrm{H}_{2} \mathrm{O}\right)_{8}\right]+++$, and the nearness of its characteristic band to that of $\left[\mathrm{Cr}(\mathrm{en})_{3}\right]^{+++}$is noteworthy. When a solution of chromic sulphate is boiled, sulphate ions displace water molecules from the complex and the green colour is thought to be due to $\left[\mathrm{Cr}\left(\mathrm{H}_{2} \mathrm{O}\right)_{4} \mathrm{SO}_{4}\right]^{+}$. We find the characteristic band of the green solution appears, much broadened, at about $6820 \mathrm{~A}$., which is in qualitative agreement with this point of view. Similar results have also been obtained with green solutions of chromic chloride in which one or more chloride ions have entered the co-ordination sphere.

We consider that the bonds uniting ethylene diamine and oxalate radicles to chromium are pre. dominantly, though not purely, covalent, since all these complex ions can be resolved into pairs of optical isomers ${ }^{2}$. The presence of extremely narrow bands in their spectra is at first sight surprising, since such bands are usually associated with electron transitions in free ions, as for example, those of certain rare earth metals ${ }^{3}$. However, the phenomenon is quite in harmony with Linus Pauling's 'model' of the molecular ion 4 , for even when co-ordinated, chromic ion possesses three unpaired electrons and it may be supposed that the absorption of a quantum of red light produces an excited state in which the spin moments of two of the electrons cancel one another, the multiplicity being thereby reduced from four to two. Thus arises the possibility of pure electron transitions in a covalently linked molecular ion. On the other hand, the co-ordination compounds of cobaltic ion contain no unpaired electrons and no unoccupied $3 d$ levels, so electron transitions of the type described above are cloarly impossible. Certainly we have been unable to discover any narrow bands in the absorption of $\left[\mathrm{Co}(\mathrm{en})_{3}\right]+++$, $\left[\mathrm{Co}\left(\mathrm{C}_{2} \mathrm{O}_{4}\right)_{3}\right] \cdots$ or $\left[\mathrm{Co}(\mathrm{CN})_{6}\right] \cdots$ within the visible spectrum.

\section{Department of Chemistry, University, Bristol. Feb. 7. \\ 1 J. prakt. Chem., 47, 305; 1893. \\ a Johnson, Trans. Far. Soc., 28, $845 ; 1932$. \\ 4 J. Amer Chedding, Phys. Rev., 34, 945; 1929}

C. H. Johnson.

A. MEAD.

\section{Mycorrhiza in the Genus Citrus}

RECENT experiment points to a close and direct correspondence between the incidence and character of mycorrhizal infection and differences in the rooting medium. In view of the existence of mycorrhiza as 